\title{
Single top-quark production cross-section using the ATLAS detector at the LHC
}

\author{
Irina Cioară, On behalf of the ATLAS Collaboration \\ Physikalisches Institut, University of Bonn (DE) \\ E-mail: irina.cioara@cern.ch
}

\begin{abstract}
Measurements of single top-quark production in proton-proton collisions are presented based on the $8 \mathrm{TeV}$ and $13 \mathrm{TeV}$ ATLAS datasets. In the leading-order process, a $W$ boson is exchanged in the $t$-channel. The fiducial cross-sections measurements for the production of single top-quarks and single anti-top-quarks, as well as the total cross-sections and their ratios are presented. At $8 \mathrm{TeV}$, differential cross-section measurements of the $t$-channel process are also reported, these analyses include limits on anomalous contributions to the $W t b$ vertex and measurement of the top quark polarization. A measurement of the production cross-section of a single top quark in association with a $W$ boson, the second largest single-top production mode, is also presented. Finally, evidence for $s$-channel single-top production in the $8 \mathrm{TeV}$ ATLAS dataset is presented. All measurements are compared to state-of-the-art theoretical calculations.
\end{abstract}

25th International Workshop on Deep Inelastic Scattering and Related Topics 3-7 April 2017

University of Birmingham, Birmingham, UK 


\section{Introduction}

With its unprecedented reach in centre-of-mass energy and luminosity, the Large Hadron Collider has proved to be a remarkable laboratory for the study of the heaviest particle predicted by the Standard Model (SM), the top quark. Millions of top quarks have been produced so far in collisions recorded by the ATLAS detector [1], opening the possibility of studying processes with relatively low cross-sections, such as different channels of single top-quark production.

The production of a single top-quark occurs via electroweak charged current interaction. At leading order QCD this is possible through one of three mechanisms. A virtual $W$ boson can be exchanged in the $s$ - or $t$-channel or the top quark is produced in association with a real $W$ boson. Measurements of any of these three productions channels are excellent probes of the $W t b$ coupling. Additionally, these processes offer the possibility of studying properties of the top quark in both its production and decay.

The dominant production mechanism is the $t$-channel exchange. For $8 \mathrm{TeV}$, at NLO precision in QCD, the predicted cross-section is $\sigma_{\text {theory }}^{\text {t-chanel }}(t q)=54.9_{-1.9}^{+2.3} \mathrm{pb}$ and $\sigma_{\text {theory }}^{\text {t-channel }}(\bar{t} q)=29.7_{-1.5}^{+1.7} \mathrm{pb}$ for the top and anti-top quark respectively. At $13 \mathrm{TeV}$ these increase to $\sigma_{\text {theory }}^{\text {t-chanel }}(t q)=136.0_{-4.6}^{+5.4} \mathrm{pb}$ and $\sigma_{\text {theory }}^{\text {t-chanel }}(\bar{t} q)=81.0_{-3.6}^{+4.1} \mathrm{pb}$ [2]. As this is the process with the largest cross-section, it is also the channel that has been studied in most detail at ATLAS, with inclusive, fiducial and even differential cross-section measurements performed at $8 \mathrm{TeV}$ [3] and $13 \mathrm{TeV}$ [4]. $t$-channel events are also used for the study of the top-quark properties.

The second largest channel at the LHC is the associated $t W$ production, representing approximately $24 \%$ of the total single-top-quark production rate at $13 \mathrm{TeV}$. The cross-section has been calculated at NLO with next-to-next-to-leading logarithmic (NNLL) soft-gluon corrections and is $\sigma_{\text {theory }}^{\mathrm{tW}}=71.7 \pm 1.8$ (scale) $\pm 3.4(\mathrm{PDF}) \mathrm{pb}[5]$.

The production mode that has the lowest cross-section is the $s$-channel production. At a centre-of-mass energy of $8 \mathrm{TeV}$, the predicted cross-section for this process is $\sigma_{\text {theory }}^{\text {s-chanel }}=5.61 \pm$ $0.22 \mathrm{pb}[6]$.

The following sections include recent ATLAS results for the measurement of all single-top production cross-sections, as well as an analysis probing the $W t b$ vertex structure and investigating top-quark properties using events produced in the $t$-channel topology.

\section{Cross-section measurements}

\section{$2.1 t$-channel production}

At $13 \mathrm{TeV}$, the inclusive measurement of the $t q$ cross-section was performed, while at $8 \mathrm{TeV}$ the fiducial, total and differential cross-sections have been measured. Both analyses looked at events with one isolated lepton, exactly two jets, one of which must be identified as coming from a $b$-quark ( $b$-tagged) and missing transverse momentum. The measurements are done separately for top and anti-top quarks, the events being sorted into these categories based on the charge of the lepton. The main sources of background are top-quark pair production $(t \bar{t})$, as well as multijet and $W$-boson production with associated jets.

An artificial neural network (NN) is used to separate signal and background and the resulting discriminant is fitted with a binned likelihood fit in order to extract the signal yields. The mea- 
surement is performed on the full $8 \mathrm{TeV}$ dataset recorded by the ATLAS detector, with respect to a fiducial region, benefiting from a strong reduction of uncertainties related to Monte Carlo (MC) generators. The fiducial phase space is defined based on stable particles at MC generator level. The measured fiducial cross-sections are $\sigma_{\text {fid }}(\bar{t} q)=5.77 \pm 0.45 \mathrm{pb}$ and $\sigma_{\text {fid }}(t q)=9.78 \pm 0.57 \mathrm{pb}$, with the main systematic uncertainties being jet energy scale, parton distribution functions (PDF) and $t q$ scale variations. The fiducial cross-sections can be extrapolated to the full phase space by correcting for the fiducial acceptance. The total cross-sections are in good agreement with the SM predictions: $\sigma_{\text {tot }}(\bar{t} q)=32.8 \pm 0.8$ (stat.) \pm 2.2 (exp.) \pm 1.7 (theo.) \pm 0.6 (lumi.) pb and $\sigma_{\text {tot }}(t q)=56.7 \pm 0.9$ (stat.) \pm 2.7 (exp.) \pm 3.0 (theo.) \pm 1.1 (lumi.) pb.

Additionally, the cross-section ratio for top and anti-top $\left(R_{t}\right)$ is measured. Due to partial cancellations of common uncertainties, this has smaller systematic errors than the respective crosssection measurements. The measured values for this ratio is $R_{t}=1.73 \pm 0.05$ (stat.) \pm 0.07 (syst.), showing no deviation from the SM expectation.

Starting from the same NN discriminant, a differential analysis is performed. The sample is enriched in $t q$ events by applying a cut and selecting events with a high NN score. Using this sample, differential cross-sections are measured as a function of the rapidity $(y)$ and $p_{\mathrm{T}}$ of the top and anti-top-quark (at particle and parton level) and $y$ and $p_{\mathrm{T}}$ of the forward jet at particle level (denoted $\hat{j}$ ). Two of the particle-level distributions are shown in Figure 1. Overall, good agreement with predictions from several MC generators is observed. As a function of the transverse momentum, the predictions seem to be somewhat harder than the data. For this measurements, the main sources of systematic uncertainties are similar to the ones discussed for the fiducial crosssection.
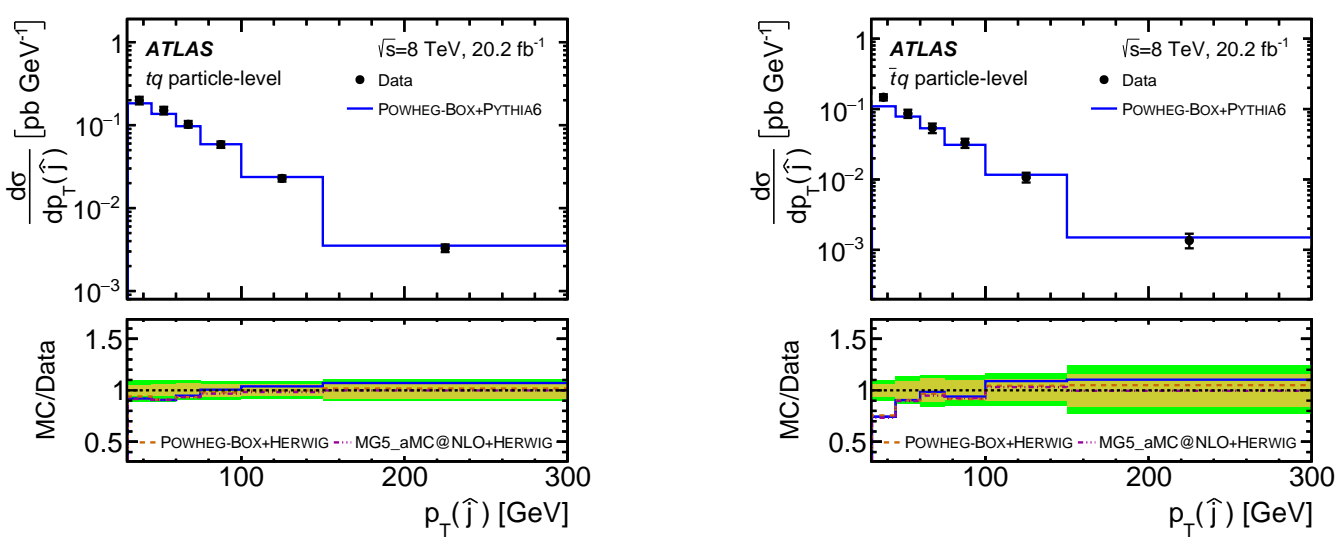

Figure 1: Absolute unfolded differential cross-section as a function of $p_{\mathrm{T}}(j)$ for top (left) and antitop quarks. The unfolded distributions are compared to various MC predictions. The vertical error bars on the data points denote the total uncertainty. The lower panel shows the MC/Data ratio and the inner (yellow) band shows the statistical uncertainty of the measurement, and the outer (green) band the total uncertainty.[3]

The analysis performed at $13 \mathrm{TeV}$, with $3.2 \mathrm{fb}^{-1}$ of data, follows a very similar strategy and measured the inclusive cross-sections and $R_{t}$. The total uncertainties amounted to $18 \%$ for events 
including anti-top quarks and $20 \%$ for top quarks. The measured cross-sections are again in very good agreement with the SM predictions.

\section{2 $t W$ production}

A measurement of the $t W$ inclusive cross-section was performed using $3.2 \mathrm{fb}^{-1}$ of data collected at a centre-of-mass energy of $13 \mathrm{TeV}$ [7]. The analysis focuses on events in which both the $W$ boson coming from the primary interaction, as well as the one resulting from the top-quark decay will produce one charged lepton. The selected final state includes exactly two opposite sign, well isolated leptons, one or two jets (out of which at least one must be $b$-tagged) and missing transverse momentum. Two distinct signal regions are then identified based on this selection (labeled $1 j 1 b$ and $2 j 1 b$ ). The main source of background is $t \bar{t}$ production. A dedicated control region enriched in $t \bar{t}$ events is defined by requiring exactly two jets that are both $b$-tagged $(2 j 2 b)$.

In order to separate the signal from the large background, boosted decision trees are trained in both signal regions. The resulting discriminants are included in a binned likelihood fit along with the events yields in the $t \bar{t}$ control region. The distributions can be seen in Figure 2. The extracted cross-section, that agrees well with the theoretical prediction, is $\sigma^{t W}=94 \pm 10$ (stat.) ${ }_{-22}^{+28}$ (syst.) \pm 2 (lumi.) pb and the result has a $4.5 \sigma(3.9 \sigma)$ observed (expected) significance. The main sources of systematic uncertainties are modelling related (due to parton shower and $t \bar{t}$ initial and final state radiation), as well as the jet energy scale.

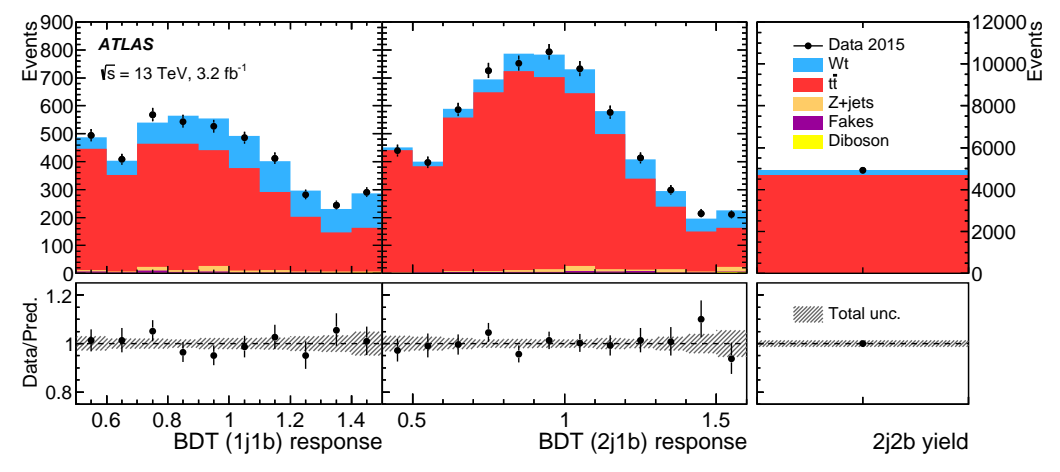

Figure 2: Post-fit distributions in the signal and control regions $1 j 1 b, 2 j 1 b$ and $2 j 2 b$. The error bands represent the total uncertainties in the fitted results. The upper panels give the yields in number of events per bin, while the lower panels give the ratios of the numbers of observed events to the total prediction in each bin.[7]

\section{$2.3 s$-channel production}

Evidence of $s$-channel single top-quark production has been reported by the ATLAS collaboration after analysing $20.3 \mathrm{fb}^{-1}$ of data collected at a centre-of-mass energy of $8 \mathrm{TeV}$ [8]. The signal region is defined by requiring exactly one isolated lepton, two $b$-tagged jets and large missing transverse momentum. The dominant background processes for this analysis are $t \bar{t}$ production and the production of a $W$ boson in association with heavy-flavour jets. In the signal region, the signal to background ratio is about $5 \%$. In order to check the $t \bar{t}$ modelling, a dedicated validation 
region is defined by applying the same selection cuts as for the signal region but requiring two additional light jets. For constraining the $W+$ jets background normalisation, a control region is defined by applying less stringent $b$-tagging criteria ( $80 \%$ working point). At least one of the selected $b$-tagged jets in that region must fail the signal region selection requirement (70\% working point).

In order to separate between the signal and background, a discriminant based on the matrix element method is used. This uses theoretical calculations to compute the probability of an event to be a signal event. The distribution of this variable in the signal region is shown in Figure 3 and is included in the binned likelihood fit. This shows a very good separation between signal and background, with the $s$-channel contribution visible in the highest bins of the distribution. In order to control the $W+$ jets background, the lepton-charge discriminant in its dedicated control region (shown in Figure 3) is also included in the fit performed to extract the final result. The total uncertainty for this measurements is $34 \%$ and is dominated by the jet energy resolution. With a $3.2 \sigma(3.9 \sigma)$ observed (expected) statistical significance, the final cross-section is measured to be $\sigma^{s \text {-channel }}=4.8 \pm 0.8$ (stat.) ${ }_{-1.3}^{+1.6}$ (syst.) pb. This result is in good agreement with the SM prediction.
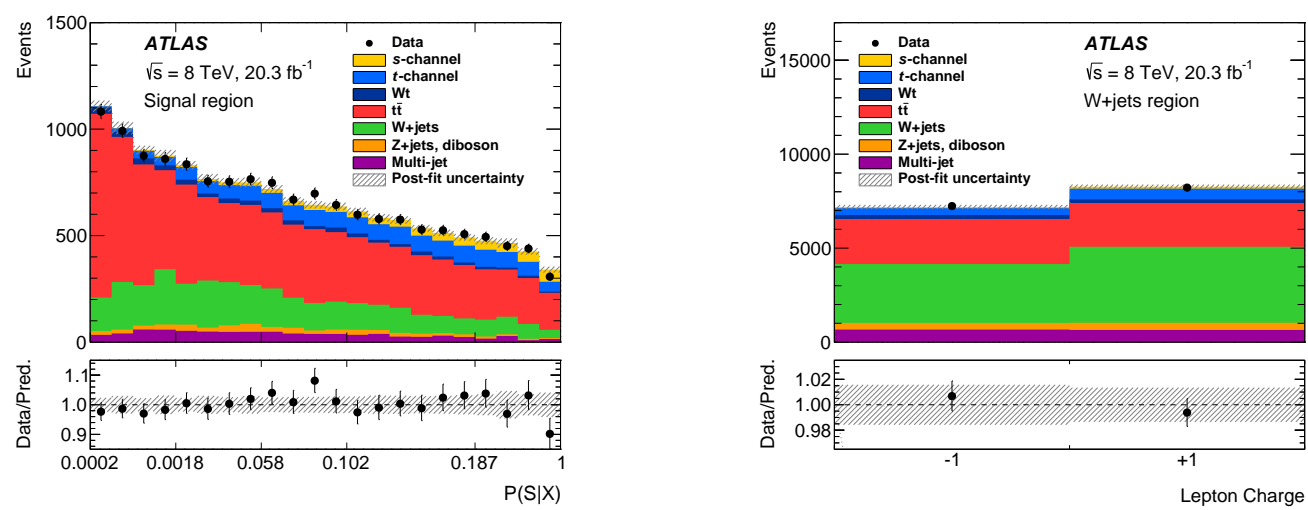

Figure 3: Post-fit distribution of the matrix element discriminant in the signal region (left) and the lepton charge discriminant in the $W+$ jets control region (right). All samples are scaled by the fit result utilising all fit parameters. The hatched bands indicate the total uncertainty of the post-fit result.[8]

\section{Top-quark properties}

In order to probe the structure of the $W t b$ vertex, several polarisation observables of the top quark and the $W$ boson were measured in $t$-channel events [9]. These observables are extracted from asymmetries in angular distributions measured at parton level. This is done by correcting for detector effects and hadronisation, after subtracting the background contributions from the $t$-channel events. The angles are measured with respect to spin quantisation axes appropriately chosen for the top quark and the $W$ boson. The measured values of the top-quark and $W$-boson polarisation observables are in agreement with the SM predictions and can be seen in Figure 4.

By combining two of the measured asymmetries (namely the normal forward-backward asymmetry and the asymmetry related to the top-quark polarisation- $A_{F B}^{N}$ and $A_{F B}^{l}$ ) and assuming $V_{L}=1$ 

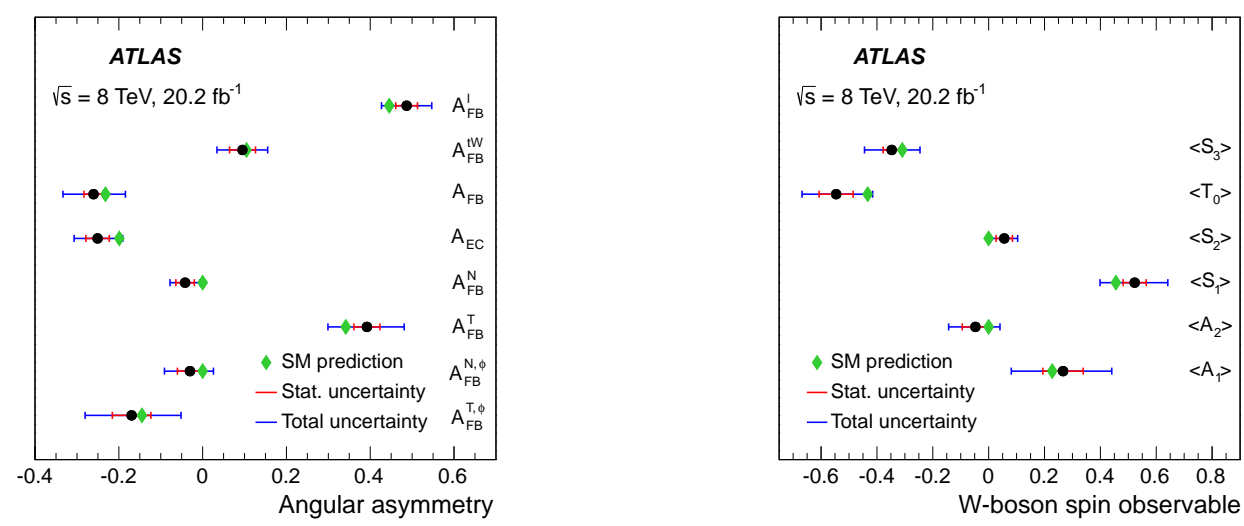

Figure 4: Summary of the measured asymmetries (left) and the measured values of the $W$-boson spin observables (right). All results are compared to the SM predictions

and that all anomalous couplings are equal to $0\left(V_{R}=g_{L}=0\right.$ and $\left.\operatorname{Re} g_{R}=0\right)$, one can set limits on $\operatorname{Im} g_{R}$. This results in $\operatorname{Im} g_{R} \in[-0.17,0.06]$, being consistent with the SM expectation.

\section{Conclusions}

With the large number of single-top quark events produced during the LHC operation so far, the ATLAS experiment has been able to measure the production cross-sections of the three different single-top channels with very good accuracy. So far, the results show no deviation from the SM predictions. Additionally, properties of the top-quark are studied in single-top $t$-channel events by looking at polarisation observables of the top quark and the $W$ boson.

\section{References}

[1] ATLAS Collaboration, The ATLAS Experiment at the CERN Large Hadron Collider, JINST 3 (2008) S08003.

[2] J. M. Campbell, R. Frederix, F. Maltoni, and F. Tramontano, Next-to-Leading-Order Predictions for t-Channel Single-Top Production at Hadron Colliders, Phys. Rev. Lett. 102 (2009) p. 182003, arXiv: 0903.0005 [hep-ph] .

[3] ATLAS Collaboration,

Fiducial, total and differential cross-section measurements of t-channel single top-quark production in pp collisions at $8 \mathrm{TeV}$ using data collected by the ATLAS detector, Eur. Phys. J. C77 (2017) p. 531, arXiv: 1702.02859 [hep-ex] .

[4] ATLAS Collaboration,

Measurement of the inclusive cross-sections of single top-quark and top-antiquark t-channel production in pp collisions at $\sqrt{s}=13 \mathrm{TeV}$ with the ATLAS detector, JHEP 04 (2017) p. 086, arXiv: 1609.03920 [hep-ex]. 
[5] N. Kidonakis, Theoretical results for electroweak-boson and single-top production, PoS DIS2015 (2015) p. 170, arXiv: 1506.04072 [hep-ph] .

[6] N. Kidonakis, NNLL resummation for s-channel single top quark production, Phys. Rev. D81 (2010) p. 054028, arXiv: 1001.5034 [hep-ph] .

[7] ATLAS Collaboration, Measurement of the cross-section for producing a W boson in association with a single top quark in pp collisions at $\sqrt{s}=13 \mathrm{TeV}$ with ATLAS, (2016), arXiv: 1612.07231 [hep-ex] .

[8] ATLAS Collaboration, Evidence for single top-quark production in the s-channel in proton-proton collisions at $\sqrt{s}=8 \mathrm{TeV}$ with the ATLAS detector using the Matrix Element Method, Phys. Lett. B 756 (2016) p. 228, arXiv: 1511.05980 [hep-ex] .

[9] ATLAS Collaboration, Probing the Wtb vertex structure in t-channel single-top-quark production and decay in pp collisions at $\sqrt{s}=8 \mathrm{TeV}$ with the ATLAS detector, JHEP 04 (2017) p. 124, arXiv: 1702.08309 [hep-ex] . 